\title{
Educação interprofissional e saúde da população LGBTQIA+: uma experiência de integração ensino-serviço-comunidade
}

\author{
Interprofessional education and health of the LGBTQIA+ population: an experience of \\ undergraduate, care and community interventions \\ Educación interprofesional y salud de la población LGBTQIA+: una experiencia de intervenciones \\ de pregrado, atención y comunidad
}

Recebido: 28/10/2021 | Revisado: 08/11/2021 | Aceito: 14/11/2021 | Publicado: 24/11/2021

Marília Pereira da Silva
ORCID: https://orcid.org/0000-0001-8397-3040
Universidade Federal do Maranhão, Brasil
E-mail: mary_silva06@live.com
Thais Regina Ferreira França
ORCID: https://orcid.org/0000-0002-2687-1985
Universidade Federal do Maranhão, Brasil
E-mail: idkthais@gmail.com
Kennedy Anderson Lobato
ORCID: https://orcid.org/0000-0003-2487-8515
Universidade Federal do Maranhão, Brasil
E-mail: kennolobatho95@gmail.com
Mariana Araujo Costa
ORCID: https://orcid.org/0000-0001-7417-8766
Universidade Federal do Maranhão, Brasil
E-mail: ma.costa@ @iscente.ufma.br
Lediane Faria da Silva
ORCID: https://orcid.org/0000-0001-8597-6747
Universidade Federal do Maranhão, Brasil
E-mail: lidisilva.faria@gmail.com
Gabrielle Silva de Carvalho
ORCID: https://orcid.org/0000-0003-3780-2376
Universidade Federal do Maranhão, Brasil
E-mail: gabriellesdcarvalho@gmail.com
Nathaly Vitória Portela Santos
ORCID: https://orcid.org/0000-0001-7212-3470
Universidade Federal do Maranhão, Brasil
E-mail: nathalyvportela@gmail.com
Carlos Eduardo Neves Amorim
ORCID: https://orcid.org/0000-0001-6183-2503
Universidade Federal do Maranhão, Brasil
E-mail: amorim.carlos@ufma.br
ORCID: https://orcid.org/0000-0003-0015-3413
Universidade Federal do Maranhão, Brasil
E-mail: sara.fiterman@ufma.br

\begin{abstract}
Resumo
Trata-se do relato de experiências de integração ensino-serviço-comunidade, de um grupo tutorial do Programa de Educação para o Trabalho em Saúde (Pet-Saúde), cujo eixo temático foi Educação Interprofissional (EIP), realizadas entre os meses de abril de 2019 a março de 2021. O projeto foi realizado em parceria entre a Universidade Federal e a Secretaria de Saúde de um município do interior do Maranhão tendo por tema a Saúde Integral da População LGBTQIA+, sendo constituído por acadêmicos de três cursos (Educação Física, Enfermagem e Medicina), preceptores da Atenção Primária em Saúde, coordenador e tutores docentes. Durante os dois anos de atividades, o
\end{abstract}


grupo pode realizar estudos relacionados as temáticas de EIP e saúde LGBTQIA+, com diagnóstico situacional e reconhecimento do território em relação a atenção dedicada a essa população. Fomentou discussões sobre as competências colaborativas e os desafios para a implantação da Política Nacional de Saúde Integral da População LGBTQIA+. Realizou pesquisas, trabalhos científicos, e confecção de artigos. Além de realizar EIP por meio do ensino remoto durante a pandemia, apoiando a comunidade e profissionais no enfrentamento da COVID-19. Concluise com a experiência do grupo PET saúde LGBTQIA+, por todo trabalho desenvolvido, que foi possível deixar um legado para universidade e para o sistema de saúde, por ter permitido aproximações antes impensadas entre cursos, estudantes, docentes, e preceptores, e por ter incentivado a implementação de discussões e reflexões sobre práticas colaborativas e especificidades da saúde da população LGBT, nunca feito antes com tal intensidade.

Palavras-chave: Educação interprofissional; Atenção à saúde; Pessoas LGBTQIA+.

\begin{abstract}
This is a report of experiences of teaching-service-community integration, of a tutorial group of the Education for Health Work Program (Pet-Saúde), whose thematic axis was Interprofessional Education (IPE), carried out between April 2019 and March 2021. The project was carried out in partnership between the Federal University and the Health Secretariat of a municipality in the interior of Maranhão with theme Integral Health of the LGBTQIA+ Population, consisting of academics from three courses (Physical Education, Nursing, and Medicine), preceptors from Primary Health Care, coordinator, and faculty tutors. During the two years of activities, the group has been able to carry out studies related to the themes of IPE and LGBTQIA+ health, with situational diagnosis and recognition of the territory in relation to the attention dedicated to this population. Promoted discussions about collaborative skills and the challenges for the implementation of the National Policy of Integral Health of the LGBTQIA+ Population. Conducted research, scientific papers, and preparation of articles. In addition to conducting IPE through remote teaching during the pandemic, supporting the community and professionals in coping with COVID-19. We conclude with the experience of the PET LGBTQIA+ Health group, for the work developed, that it was possible to leave a legacy for the university and the health system, for having allowed approximations that were previously unthought of among courses, students, teachers, and preceptors, and for having encouraged the implementation of discussions and reflections on collaborative practices and health specificities of the LGBTQIA+ population, never done before with such intensity.
\end{abstract}

Keywords: Interprofessional education; Health care; LGBTQIA+ people.

\title{
Resumen
}

Se trata de un informe de experiencias de integración docente-servicio-comunidad del grupo tutorial del Programa Educación para el Trabajo en Salud (Pet-Salud), cuyo eje temático fue Educación Interprofesional (EIP), realizado entre abril de 2019 y marzo de 2021. Establecimos una alianza entre la Universidad Federal y el Departamento de Salud de un municipio del interior de Maranhão sobre el tema Salud Integral para la Población LGBTQIA +, conformada por académicos de tres cursos (Educación Física, Enfermería y Medicina), preceptores de Salud Primaria Cuidado, coordinador y tutores docentes. Durante los dos años de actividades, el grupo realizó estudios en EIP y salud LGBTQIA +, con diagnóstico situacional y reconocimiento del territorio en relación a la atención dedicada a la población. Fomentó discusiones sobre competencias colaborativas y los desafíos para la implementación de la Política Nacional de Salud Integral para la Población LGBTQIA +. Realización de investigaciones, trabajos científicos y artículos. Además de realizar EIP mediante aprendizaje remoto durante la pandemia, apoyando a la comunidad y a los profesionales en la lucha contra el COVID-19. Se concluye con la experiencia del grupo PET salud LGBTQIA +, por todo el trabajo desarrollado, que fue posible dejar un legado para la universidad y para el sistema de salud, por los enfoques antes impensables entre cursos, estudiantes, profesores y preceptores, y por haber incentivado la implementación de discusiones y reflexiones sobre prácticas colaborativas y especificidades de la salud de la población LGBT, lo que nunca antes se había hecho con tanta intensidad.

Palabras clave: Educación interprofesional; Cuidado de la salud; Personas LGBTQIA +.

\section{Introdução}

Com a criação do Sistema Único de Saúde (SUS), emergiram mudanças na organização do trabalho nos serviços públicos, surgindo um novo desafio para formação das profissões da saúde em nosso país, pela necessidade de preparar profissionais para um trabalho coerente com seus pressupostos ético-políticos, que incluem a universalidade, a integralidade e a equidade (Santos, Simonetti \& Cyrino, 2018).

Embora a Constituição de 88 e o SUS determinem que o acesso ao sistema público de saúde seja um direito de todos, populações reconhecidas como minorias ainda enfrentam barreiras de acesso, o que inclui pessoas lésbicas, gays, bissexuais, travestis e transexuais, queers, intersexuais, assexuais e outras identidades de gênero e orientações sexuais (LGBTQIA+). 
Além disso, os problemas e necessidades de saúde atendidos no SUS tornam-se cada vez mais complexos, exigindo mudanças de paradigmas na produção de seus serviços, e colocando os usuários e suas necessidades na centralidade desse processo (Crisp \& Chen, 2014; Frenk et al., 2010).

Partindo dessas problemáticas e das evidências relativas à importância do trabalho em equipe para a melhoria da qualidade dos serviços de saúde, organizações internacionais vêm estimulando cada vez mais estratégias de formação em saúde capazes de desenvolver competências para o efetivo trabalho em equipe (Organização Mundial da Saúde, 2010).

Nesse contexto, a Educação Interprofissional (EIP) em saúde é de grande importância para o trabalho em saúde, pois ela promove um processo de aprendizado interativo e compartilhado, aprimorando a colaboração entre membros da equipe e a qualidade da assistência prestada (Stephens \& Ormandy, 2018).

A Prática Colaborativa (PC) tem por objetivo uma cooperação integrada de diferentes profissionais da área da saúde, que combinam competências e habilidades em benefício do indivíduo, com o foco na atenção centrada ao paciente, possibilitando assim melhor interação e desenvolvimento das ações de cuidado (Supper et al., 2014).

O surgimento dos debates sobre EIP e PC está diretamente relacionado com aspectos falhos dos serviços de saúde, tais como: fragmentação do trabalho, exposição do paciente a procedimentos desnecessários ou duplicados e dificuldades dos profissionais em trabalhar em equipe, manifestações de preconceito e discriminação. Tais problemáticas são resultado de um modelo primitivo de trabalho em saúde, pautado na rapidez e desintegração dos serviços, na falsa autossuficiência profissional e desconhecimento das políticas públicas de saúde (Costa, 2019).

Nesse panorama, formar profissionais com competências interprofissionais vem se tomando um desafio mundial, sendo necessário o surgimento de novas iniciativas para a formação profissional.

A criação do programa PET-Saúde/Interprofissionalidade nesse contexto, constitui-se como importante instrumento para estimular ações de interação e comunicação entre diferentes profissões. Criado em 2008 pelo Ministério da Saúde (MS) em parceria com Ministério da Educação, o programa tem induzido mudanças significativas na formação de novos profissionais e no sistema público de saúde (Magnago et al., 2019).

O incentivo das discussões e construções interprofissionais tem perpassado não somente por problemas clássicos do sistema de saúde como um todo, mas também pela assistência à saúde de populações específicas e mais vulneráveis, onde a saúde da população LGBTQIA+ tem conquistado espaço importante nas discussões. Por outro lado, muitos profissionais e acadêmicos, da saúde não se sentem qualificados o suficiente para o cuido com essa população. São raras as situações em que as temáticas sobre gênero, orientação e identidades sexuais, fazem parte da rotina do atendimento profissional ou são foco do ensino-aprendizagem. Por isso, é necessário reforçar a importância desse debate para formação, reorganização dos serviços e para assistência dos usuários de saúde (Da Costa et al., 2017). Logo, a integração ensino-serviço-comunidade são fundamentais para garantir a sensibilização e qualificação dos profissionais e acadêmicos da saúde acerca das particularidades LGBTQIA+e o efetivo cumprimento do direito à saúde (Mello et al., 2011). Com isso, a EIP tem o papel de fortalecimento no SUS e no atendimento em saúde como um todo.

Assim, o presente artigo vem relatar as experiências de integração ensino-serviço-comunidade, de um grupo tutorial do Programa de Educação para o Trabalho em Saúde (Pet-Saúde), voltado para a temática de Saúde Integral da População LGBTQIA+.

\section{Metodologia}

Trata-se do relato de experiências de integração ensino-serviço-comunidade vivenciadas pelo grupo Pet-Saúde LGBTQIA+, do Projeto PET 38, que foi fruto de parceria entre a Universidade Federal do Maranhão (UFMA), Campus Pinheiro e a Secretaria Municipal de Saúde de Pinheiro (Estrela, 2018; Pereira et al., 2018; Koche et al., 2011; Ludke \& Andre, 
2013).

O PET-Interprofissionalidade (PET EIP) foi um programa do Ministério da Saúde em parceria com o Ministério da Educação, que objetivou a qualificação dos processos de integração ensino-serviço-comunidade, de forma articulada entre o Sistema Único de Saúde-SUS e as instituições de ensino, de modo a promover a Educação Interprofissional-EIP e as Práticas Colaborativas em Saúde (Brasil, 2018).

Para compor o PET EIP, houve processo seletivo por meio do Edital MS-SGTES 10/2018, do qual resultaram 120 projetos aprovados, dentre os quais o projeto 38, que desenvolveu suas atividades por dois anos, através de cinco grupos tutoriais temáticos (saúde LGBTQIA+; imunização; terapias integrativas; hanseníase; saúde da mulher).

Uma vez aprovado o projeto, seguindo as determinações do Edital supracitado, os interessados tiveram que passar por um processo seletivo interno. Em relação aos acadêmicos, foram incluídas vagas para três cursos de saúde presentes no campus Pinheiro (Educação Física, Enfermagem e Medicina). Para estes, as condições da seleção envolviam, estar entre $1^{\circ}$ e o $5^{\circ}$ períodos do curso, nota de prova escrita e coeficiente de rendimento acadêmico. Coordenadores de grupo e tutores, deveriam ser professores de graduação em exercício da docência, envolvidos com processo de mudança curricular e de integração ensino-serviço-comunidade, com ênfase no desenvolvimento de iniciativas interprofissionais. Os preceptores, por sua vez foram indicados pela SEMUS, devendo ter nível superior de formação, atuantes e vinculados à gestão ou à atenção do SUS, envolvidos em atividades de integração ensino-serviço-comunidade no município. Para estes, a seleção se deu por carta de intenção, entrevista e currículo. Todos os participantes, tanto bolsistas, quanto voluntários, passaram por processo seletivo, que foram realizados 4 vezes, para reposição de participante que precisaram sair durante os dois anos de projeto.

As atividades do projeto 38 aconteceram entre os meses de abril de 2019 a março de 2021, e foram voltadas para promoção da integração ensino-serviço-comunidade com foco no desenvolvimento do SUS a partir dos elementos teóricos e metodológicos da EIP, em associação com as questões temáticas de cada grupo, sendo as intervenções do grupo saúde LGBTQIA+, objeto deste manuscrito.

Destaca-se ainda que toda produção do PET 38, obedeceu aos princípios éticos determinados na Resolução CNS 466/99 e está aprovada no Comitê de Ética em Pesquisa da Universidade Federal do Maranhão sob o CAAE nº 29652520.0 .0000 .5087$.

\section{Relato de Experiência}

Inicialmente, vale destacar que o tema de Saúde LGBTQIA+ já vem sendo trabalhado no campus de Pinheiro, por meio de atividades de extensão. Assim, ao elaborar o projeto para seleção do PET EIP, o tema entrou como eixo temático nos grupos tutoriais.

O grupo de saúde LGBTQIA+, ao longo de todo projeto, desenvolveu uma trajetória de imersão nos referenciais teóricos da EIP, e de questões de saúde e diversidade de gênero, seguida de intervenções colaborativas para inserção destes temas na graduação, nos serviços de saúde e na comunidade. Logo, abordou a Atenção Integral a Saúde da População LGBTQIA+ na APS alinhada à EIP e às PC, através de estratégias que possibilitassem melhor relação no ambiente profissional e acadêmico, questionamentos e conhecimento sobre a saúde LGBTQIA+, facilitação do processo do trabalho em equipe, comunicação e resolução de conflitos, proporcionando dia após dia uma rede de cuidado mais preparada, acolhedora e humanizada para essa população.

Entre os participantes do grupo, tivemos dois coordenadores, quatro tutores, 5 preceptores e 21 alunos, que entre ingresso e saída, se alternaram nas seguintes vagas: 01 coordenador bolsista, 01 tutor bolsista, 01 tutor voluntário, 03 preceptores bolsistas, 06 alunos bolsistas e 06 alunos voluntários. A dedicação em horas semanais era de 8h para todos os participantes, e as horas eram divididas em atividades intragrupo e intergrupos, na IES, na rede e nos territórios. 


\section{Compreendendo a interprofissionalidade, e a saúde LGBTQIA+ nos espaços de ensino, serviço e comunidade}

As atividades do primeiro semestre iniciaram em abril de 2019, e estiveram voltadas para: (1) estudos relacionados as temáticas de EIP e saúde LGBTQIA+; (2) diagnóstico situacional das unidades de saúde e reconhecimento do território.

Inicialmente as atividades envolveram participação em curso on-line sobre Educação Interprofissional em Saúde, ofertado pela UFRN, leitura e fichamento da Política Nacional de Saúde Integral LGBTQIA+, com rodas de conversa sobre sua aplicação, e de artigos que abordavam as temáticas de EIP e diversidade de gênero, com discussões sobre o conteúdo.

Os participantes prestigiaram a webpalestra Atendimento à População LGBTQIA+ na Atenção Primária à Saúde ofertada pelo telessaúde da UFRGS, e as gravações de quatro palestras sobre interprofissionalidade do workshop Universidade e Poder Público, da USP Bauru, com realização de resumos individuais sobre os materiais.

Para ampliação dos conhecimentos, o grupo assistiu filmes e documentários que abordavam a temática como: De gravatas e unhas vermelhas; Bombadeira; Uma Mulher Fantástica; playlist de vídeos Vivências LGBTQIA+. Todos com rodas de discussão sobre as questões abordadas.

Realizou-se ainda um diagnóstico situacional da atenção à essa população, com visitas a diferentes espaços, envolvendo três unidades de saúde, com aplicação de questionário sobre conhecimentos relacionados a interprofissionalidade e política de saúde integral LGBTQIA+, além da construção de uma matriz SWAT (FOFA).

Visitou-se também delegacias, para ter acesso aos registros de boletins de ocorrência que permitissem conhecer os casos de violências contra as pessoas LGBTQIA+, e no território, fez-se um levantamento de pontos locais de apoio a esta população, como ONG'S, grupos organizados, e outros atores sociais.

Nesta etapa de diagnóstico situacional, procedeu-se ainda pesquisa e análise das condições de saúde dos territórios de abrangência do projeto, e investigação da situação epidemiológica do Município em relação a população LGBTQIA+.

Tudo era novo para os participantes. Dos temas discutidos, da relação entre profissionais da rede de saúde, alunos e docentes de diferentes cursos, até o pensar coletivo sobre o ensino e extensão. Entretanto, cumpre destacar que o grupo tinha encontros semanais, onde discutiam as suas atividades, mantendo sempre decisões colegiadas.

Dessa imersão no campo e dos diálogos do grupo com profissionais, população e entre os participantes, observou-se que apesar dos avanços políticos e sociais, ainda persiste uma invisibilização das necessidades específicas dessa população. Percebeu-se uma ausência de registros específicos em bancos de dados da saúde, ausência de aulas sobre saúde LGBTQIA+ nos cursos de graduação envolvidos, que delegacias da cidade não realizavam queixas por orientação sexual, e que as unidades de saúde não possuíam atendimento específico e não usavam o nome social. Além disso, embora tenhamos buscado grupos sociais voltados para organização dessa população, só encontramos informações de pessoas que isoladamente já haviam se pronunciado em relação a direitos e necessidades LGBTQIA+. Assim, o grupo elaborou, colaborativamente, questionários para pesquisa sobre percepção quanto ao acesso à saúde pela população LGBTQIA+, bem como gestores de saúde, gerentes e profissionais das unidades básicas de saúde do município.

Nos espaços da saúde, foram identificadas ainda dificuldades para atendimentos dos usuários LGBTQIA+, pois os profissionais, embora estando diretamente envolvidos no cuidado dessa população, não possuíam conhecimento e formação para uma assistência adequada e em acordo com o preconizado, e relataram ainda dificuldades locais para a implantação da Política Nacional de Saúde Integral da População LGBTQIA+ (que envolviam a população em geral e LGBT, processos de trabalho e gestão). Diante disso, o grupo realizou discussões sobre como iniciar as atividades sem provocar resistências.

Sabe-se que existe um preconceito institucional em muitos serviços sanitários, o que prejudica não apenas a qualidade do atendimento, mas a própria saúde das pessoas LGBTQIA+. A LGBTfobia representa de forma genérica todo o conjunto de atitudes que envolvem aversão, preconceito, violência ou discriminação contra a comunidade LGBTQIA+. Conhecer essa condição é necessário, uma vez que, no campo da construção social dos corpos, a sexualidade não é um fenômeno isolado, mas 
pertence às dinâmicas de posições e oposições que organizam toda a sociedade e ditam normas e regras a serem seguidas (Junqueira, 2007). Como apontam Vitiritti, Andrade e Peres (2016), os agentes do campo da saúde, antes de serem profissionais, são participantes de grupos sociais influenciados por modelos culturais. Esses agentes carregam uma bagagem cultural, histórica e social construída com base no modelo hegemônico de heteronormatividade e cissexismo. Tal bagagem pode se refletir na exteriorização de sentimentos e atitudes negativas ante a população LGBTQIA+, o que implica deficiências na assistência prestada.

Das rodas de conversas do grupo, emergiu também por parte de alguns participantes, fragilidades nos conhecimentos em relação as questões de diversidade sexual e de gênero, e da assistência à saúde desta população. Foi então incluída na programação aulas expositivas: (1) sobre Política Nacional de atenção integral a saúde LGBTQIA+, (2) terminologias no contexto LGBTQIA+, seguidas de debates, e ainda a uma oficina sobre metodologias e tecnologias na educação em saúde com a elaboração de construção de material ilustrativo de apoio as capacitações. Assim, foram confeccionados um manual de orientação para produção audiovisual, e um manual de comunicação LGBTQIA+.

Silva et al (2020) enfatizam a necessidade da inclusão desta temática nos currículos da saúde, para que todos os profissionais da saúde tenham o conhecimento dos fundamentos necessários para proporcionar acolhimento, prevenção, promoção e terapêutica de qualidade à população LGBTQIA+.

Outro ponto relevante observado pelo grupo, foram as dificuldades para exercer interprofissionalidade, uma vez que docentes, estudantes e profissionais de diferentes formações, dividiam o mesmo ambiente institucional, mas não possuíam hábitos de interação e diálogo. O PET trazia para os participantes essa oportunidade, mas era necessário extrapolar os muros do projeto e levar essa experiência para a comunidade acadêmica e profissionais de saúde da rede.

A interprofissionalidade favorece as trocas de informação e conhecimento, a cooperação solidária nos fazeres, a atenção corresponsável às necessidades em saúde para a construção de projetos terapêuticos e de promoção da saúde, o agir coletivo em território e a rede de laços afetivos que dá mais intensidade ao pertencimento a uma equipe (Ceccim, 2018).

Percebeu-se ainda, em relação a EIP, a relevância dos conceitos de integração e intencionalidade para fazer dar certo as ações do projeto.

Dessa forma, ao término do semestre realizou-se uma oficina para reavaliar objetivos e metas do projeto como um todo. Era possível observar que as atividades instrumentalizaram os participantes no tocante ao conhecimento sobre as temáticas estudadas, e permitiram uma aproximação singular entre a universidade e a rede de saúde. Assim, fomentaram a reorganização das ações de integração ensino-serviço-comunidade no território, e permitiram pensar ações na graduação em consonância com as reais necessidades em saúde da população LGBTQIA+ do município.

Estimulando o desenvolvimento de competências colaborativas e atenção à saúde da população LGBTQIA+ em diferentes espaços.

No segundo semestre, as atividades de estudo sobre as temáticas EIP e saúde LGBTQIA+ foram mantidas, mas o grupo pode realizar ações em integração com o ambiente acadêmico em geral (outros docentes e discentes que não integravam o PET), e com as unidades de saúde, para levar informações e promover reflexões.

As competências colaborativas passavam a ser reconhecidas como um pilar para as ações do grupo, e as atividades sempre envolviam uma intencionalidade para mantê-las presentes. Dessa maneira, após a fase inicial de capacitações, o novo semestre começava com oficinas para a comunidade acadêmica e profissionais de saúde sobre as competências colaborativas.

A equipe multidisciplinar precisa conduzir suas práticas para reflexões conjuntas, em que a colaboração da equipe possa reconhecer as lacunas na perspectiva do trabalho interprofissional e que, efetivamente, haja uma interpenetração das 'disciplinas' e integração entre as práticas profissionais, auxiliando o processo de construção da interprofissionalidade na saúde (Farias et al., 2018). 
Pensando em possíveis ações de EIP, o grupo se envolveu na organização de atividades com simulação clínica (encomendas e estações), e aprendizagem baseada em problemas (confecção de caderno de casos de saúde LGBT+ para PBL), para subsidiar ações educativas voltadas para a comunidade acadêmica e para profissionais de saúde da rede.

Para se aproximar e levar conhecimentos à comunidade acadêmica, e população em geral, o grupo tutorial se envolveu em eventos acadêmicos como o MUSAS II (Mulheres na Atenção à Saúde - $2^{\mathrm{a}}$ Edição) onde inseriu temas sobre saúde da mulher trans por meio de simulação, e Feira das Profissões na UFMA, para trabalhar a clareza de papeis.

O semestre também foi marcado pelo movimento de inserção de atividades nos serviços de saúde, incluindo rodas de conversa, ações educativas e de sensibilização sobre conceitos fundamentais de interprofissionalidade e saúde da população LGBTQIA+. Inclusive, ofertou-se um curso presencial de 06 horas destinado aos profissionais de saúde (médicos, enfermeiros, odontólogos, profissionais do Núcleo de Apoio à Saúde da Família - NASF e Agentes comunitários de saúde) sobre a EIP e a Política Nacional de Saúde Integral da População LGBT em uma Unidade Básica de Saúde elegida para aplicação da ação.

O PET-Saúde constitui-se uma estratégia de indução de mudanças no processo de formação profissional, possibilitando a identificação de necessidades frequentes vivenciadas no serviço de saúde e a reflexão por parte dos profissionais, a fim de originar propostas de intervenção contínuas (Almeida et al., 2019).

Realizamos já quase ao final do semestre um workshop de criatividade, onde convidamos toda comunidade acadêmica para assistir apresentações com as atividades realizadas por cada grupo, incluindo momentos de comentários e sugestões, e participação em diferentes estações simuladas.

Assim, a simulação clínica, foi uma estratégia de ensino aprendizagem que também subsidiou o trabalho de EIP no grupo, utilizando uma estação sobre paciente com necessidade de transfusão, onde a doadora era uma irmã trans destratada por profissional de saúde. Aplicada na universidade para toda comunidade acadêmica e nas unidades de saúde para preceptores e demais profissionais, pudemos trabalhar as competências colaborativas (trabalho em equipe, resolução de conflitos, clareza de papéis e comunicação interprofissional), sendo o momento do debriefing, de grande relevância para reflexões sobre a homofobia em serviços de saúde, na comunidade e também sobre a interprofissionalidade.

Para Almeida et al (2019) é importante o fortalecimento da interação entre ensino, serviço e comunidade, extinguindo a cultura do ensino fragmentado e do trabalho individualizado. O despreparo de profissionais de saúde para lidar com questões relacionadas com a diversidade sexual e de gênero constitui uma importante barreira tanto para o acesso aos serviços quanto para o cuidado integral à saúde da população LGBTQIA+ (Bezerra et al., 2019).

Nesse sentido, de acordo com Rohrs et al (2017) a simulação realística desperta maior interesse dos discentes, ao ser uma metodologia de ensino ativa que permite uma aproximação com a realidade e possibilita cometer erros, debater as ações e efetuar um atendimento de qualidade, em que no contexto da realidade seria algo ainda limitado.

Faz-se necessário conhecer e reconhecer as vulnerabilidades e especificidades desta população para apontar as demandas no campo da saúde da população LGBTQIA+ bem como avaliar se seus objetivos foram alcançados (Prado \& Sousa, 2017).

Confeccionar materiais didáticos para disseminação de conhecimentos, foi eleito pelo grupo como importante instrumento para inserção de conhecimentos sobre interprofissionalidade, prática colaborativa e política de atenção à saúde integral da população LGBTQIA+. Dessa forma, a fim de fornecer as informações essenciais de maneira objetiva, simples e acessível, foram confeccionados cartazes sobre: (1) a necessidade de evitar discriminação quanto a orientação sexual e identidade de gênero no SUS; (2) sobre a Política de Atenção Integral à Saúde da população LGBTQIA+; (3) autodeterminação de pessoas trans e travestis através do enfoque no uso do nome social, com a frase Aqui você pode usar o nome social; (4) educação interprofissional e as competências colaborativas (todos colocados nos corredores da universidade e/ou serviços de saúde), além de vídeos animados de curta duração com estas informações, para circulação em redes sociais. 
Outra atividade interessante, foi uma avaliação qualitativa do primeiro ano do projeto, onde utilizamos a metodologia de foto relato. Todos os participantes foram convidados a escolher uma foto que representasse sua percepção sobre a experiência vivida, e em uma reunião, compartilhar a foto e narrativa sobre o motivo de sua escolha e sua opinião sobre o primeiro ano de projeto. Das falas, surgiram diversos pontos positivos em relação a integração ensino serviço e comunidade, sobre a produção de conhecimentos, sobre a aproximação entre os participantes, sobre a relevância do trabalho interprofissional, e sobre as mudanças pessoais e organizacionais provocadas pela experiência. Surgiram também os pontos negativos, como as dificuldades na organização de horários para os encontros presenciais e atividades, ausência de apoios institucionais, e resistência em diversos espaços em relação a temática (mas tais resultados serão objeto de outro artigo). Como sempre, o semestre encerrava com uma revisão do planejamento e adequação das atividades.

\section{Desafio de trabalhar com interprofissionalidade, e a saúde LGBTQIA+ frente ao distanciamento social}

O terceiro semestre do projeto iniciava com grandes expectativas, conforme já mencionado, havia-se realizado nova discussão sobre o planejamento das atividades, e com as experiências vividas o grupo tinha traçado novos planos e pretendia aumentar a aproximação com a rede de saúde, trabalhar com possibilidades de inserção dos conteúdos nos currículos dos cursos, promover eventos científicos no espaço acadêmico, e se aproximar da população LGBTQIA+ do município.

Já de início, realizou-se uma ação educativa em escola municipal para alunos do ensino médio, sobre puberdade, educação sexual; métodos contraceptivos e gravidez na adolescência. Seguindo a programação do projeto como um todo, onde foi ofertada uma oficina sobre competências colaborativas, o grupo organizou a formação em dois momentos, onde foi apresentado uma simulação sobre gestão de conflitos e comunicação interprofissional, posteriormente ofertado uma aula expositiva sobre os respectivos temas.

Em associação aos demais grupos tutoriais, realizamos uma recepção para os calouros dos cursos da saúde apresentando a proposta do PET e ações de cada grupo tutorial. Por ocasião, os grupos organizaram uma atividade colaborativa. No grupo LGBT+, convidou-se os calouros a construir um espaço de vivências no campus, com almofadas nas cores do arco-íris, murais com mensagens, fotos e muito acolhimento.

Logo em seguida, as aulas foram suspensas por tempo indeterminado devido a chegada ao país de casos de infecção pelo novo coronavírus, denominado SARS-CoV-2, causador da doença COVID-19. Era um momento difícil para o mundo inteiro, mas as atividades do projeto, por decisão conjunta dos participantes, não pararam. Foi desafiador tomar decisões, trabalhar e interagir quando a ordem era se distanciar para segurança de todos, mas a via digital, com suas tecnologias da informação e comunicação, plataformas virtuais de aprendizagem, redes sociais foi se apresentando como uma potente ferramenta, propulsora da criação de novas relações interpessoais e educativas. Através dela conseguimos manter as ações do último ano do projeto. As reuniões semanais passavam a acontecer por meio de plataformas digitais, e o planejamento foi sendo discutido e revisto mês a mês, pois não existia previsão de retorno as atividades presenciais.

Neste contexto, em relação a indução de mudanças no espaço acadêmico, os participantes fizeram a leitura para compreensão dos PPCs dos cursos envolvidos no projeto, com o desenvolvimento de proposta de ementa para construção de disciplina optativa sobre saúde integral da população LGBT (sua inserção está sendo discutida no novo PPC de medicina, com proposta em criação), bem como a proposta de integração da temática em disciplinas já existentes, por meio do caderno de casos de saúde LGBT+ para PBL. Além disso, conseguimos inserir docentes participantes do PET nos núcleos docentes estruturantes dos três cursos.

As atividades de simulação foram adaptadas, criando-se o PETFLIX, onde exibíamos episódios da série unidade básica, produzida pela globoplay, como a simulação da rotina dos serviços da saúde, e seguíamos para o debriefing discutindo a interprofissionalidade e as competências colaborativas. 
Para indução de mudanças no trabalho em saúde, passamos a realizar alguns webinários, envolvendo questões LGBT+ e ainda de outras populações que enfrentam da mesma feita, dificuldades no acesso a saúde: (1) Saúde Mental LGBTQIA+ \& Isolamento Social; (2) População Negra \& COVID-19; (3) Saúde Indígena e COVID-19; (4) Uso de Álcool \& Drogas, Pandemia \& Populações Vulneráveis; (5) Saúde da População LGBTQIA+ no Contexto da Atenção Primária; (6) Libras e atendimento à saúde da pessoa com deficiência auditiva. Nestas atividades, investiu-se na divulgação e conseguimos uma audiência de outras universidades e outros municípios e estados.

A produção de materiais educativos, outra estratégia importante, passava a incluir orientações quanto aos cuidados com a COVID-19, e dessa forma o grupo realizou estudos remotos de materiais sobre comunicação de notícias difíceis, e protocolo SPIKES voltados para a COVID-19 e a população LGBT, participou de minicurso de $4 \mathrm{~h}$, doenças ocasionadas por vírus respiratórios emergentes, incluindo a COVID-19 (EaD) ofertado pela escola de governo do ministério da saúde, e do curso de 20h, sobre relacionamento interpessoal, ofertado por Instituto Nacional de Ensino a Distância. Os participantes foram estimulados a produzirem materiais educativos autoinstrucionais como: (1) vídeos educativos sobre comportamentos e atitudes que protegem dos riscos de contágio da COVID-19; (2) podcast Impactos socioculturais no atendimento da APS em Pinheiro; (3) panfleto digital sobre comunicação de notícias difíceis no contexto da pandemia; (4) caderno digital sobre enfrentamento da COVID 19; (5) Caderno de propostas de Intervenção para a rede de Saúde de Pinheiro, MA no contexto da pandemia da COVID-19, encaminhado para secretaria municipal de saúde; (6) participação em rodas de conversa com os profissionais de saúde do município para discutir sua saúde mental em tempos de pandemia, que foram idealizadas pelo grupo de imunização, onde contamos com dois psicólogos.

Neste período, o grupo também se envolveu em produção científica, pois em decisão do grupo colegiado condutor do projeto 38, todos os participantes teriam que participar de atividades de ensino, pesquisa e extensão. Assim, foram elaborados e aplicados questionários de avaliação dos impactos do projeto, apresentados trabalhos em eventos científicos, escritos artigos sobre as experiências do grupo e com a coleta de dados realizada. Seguimos ainda com duas grandes propostas idealizadas para o projeto, sendo um e-book sobre interprofissionalidade, no qual escrevemos um capítulo sobre Gestão de Conflitos e Comunicação Interprofissional, e um evento científico que deveria ser local e presencial, mas que acabou sendo nacional e online, que foi o I Encontro Nacional de Educação Interprofissional de Saúde, onde tivemos mais de 1.000 inscritos, 20 palestrantes com grande produção científica no assunto, e onde o grupo compôs a comissão científica e organizou os anais, junto ao grupo de práticas integrativas.

A EIP visa à construção de conhecimento no coletivo por meio do diálogo e do respeito às diferenças inerentes em um grupo de profissionais, no que diz respeito ao exercício do trabalho e do núcleo de saber de cada um. Ganham destaque as consultas compartilhadas, discussões de casos e atividades grupais (Vendruscolo et al., 2020).

Pereira (2018) relata que o trabalho interprofissional em Saúde pode ser mais aderente, compartilhado, colaborativo e seguro, portanto, mais ecológico, sendo, assim, mais prazeroso, menos insalubre, integrado e com maior reciprocidade, pode ampliar a fixação dos profissionais, com territorialização de suas práticas, permitindo avançar em uma ação planejada e avaliada pela equipe, com maior eficácia e efetividade na organização do processo de trabalho, no cuidado integral e na educação permanente da equipe.

Ao final do projeto, realizamos uma avaliação quantitativa por meio de formulário eletrônico, e qualitativa, por meio de roda de conversa virtual, sendo nos dois espaços a experiência vivida considerada pela grande maioria como relevante para melhorias na formação e na assistência à saúde.

Realizar EIP por meio do ensino remoto, educação a distância e educação online, em tempos de pandemia, foi um desafio que enfrentamos graças as potencialidades do uso das tecnologias digitais em rede, da criatividade dos participantes, e principalmente da relação dialógica e colaborativa que construímos ao longo do projeto. 


\section{Considerações Finais}

Ao término do projeto, é notória a necessidade de abordar o atendimento à população LGBTQIA+ na formação em saúde, desde o ensino escolar, graduação, pós-graduação e educação permanente, para minimizar a invisibilidade na qual os serviços persistem em manter as particularidades do contexto de necessidades, saúde e vida dessa população. Da mesma forma a inclusão da interprofissionalidade, para incentivar a aproximação entre profissões, e o trabalho colaborativo em equipe, redirecionando o modelo separatista de formação em saúde, e possibilitando desenvolvimento de ações de cuidado mais oportunas para o enfrentamento da complexidade dos problemas que se apresentam ao SUS.

Projetos como o PET saúde, que incentivam a inserção da EIP numa proposta ensino-serviço-comunidade, são de grande relevância, pois incentivam parcerias entre os espaços acadêmico e de gestão pública da saúde, para indução de mudanças na formação e nos serviços, com grande potencial para dar sustentação a ações integrais e mais resolutivas para assistência em saúde.

A existência de bolsas, também se mostrou relevante, principalmente por se tratar de curso da expansão, localizado em pequeno município, a realidade local costuma não ser tão facilitadora de tais práticas. Embora o trabalho não se limite ao suporte financeiro, é indiscutível que este amplia interesses, e incentiva maior envolvimento de docentes, discentes e preceptores, com práticas educativas e assistenciais da extensão.

A experiência do PET saúde LGBTQIA+, por todo trabalho desenvolvido, deixa um legado para universidade e para o sistema de saúde, seja por ter permitido aproximações antes impensadas, entre diferentes cursos, estudantes, docentes e preceptores, por ter incentivado a implementação de discussões e reflexões sobre um olhar específico em relação a saúde dessa população, nunca feito antes com tal intensidade, ou até mesmo pelo volume de materiais autoinstrucionais produzido, e mudanças ambientais realizadas em prol de maior inclusão.

Entretanto promover saúde à população LGBTQIA+ em uma equipe interprofissional ainda é um desafio para o sistema público de saúde, o que nos faz concluir pela necessidade de outros investimentos governamentais para manutenção de trabalhos para incentivo de EIP e inserção das premissas básicas da Política de Atenção Integral à Saúde da população LGBT. A realização de novos trabalhos com a mesma temática ampliando as buscas se faz necessário para que possamos entender as repercussões da EIP não somente na atenção básica, mas em todos os setores do Sistema Único de Saúde no suporte e na assistência à saúde da população LGBTQIA+.

\section{Referências}

Almeida, R. G. dos S., Teston, E. F., \& Medeiros, A. de A. (2019). A interface entre o PET-Saúde/Interprofissionalidade e a Política Nacional de Educação Permanente em Saúde. Saúde em Debate, 43(spe1), 97-105.

Bezerra, M. V. da R., Moreno, C. A., Prado, N. M. de B. L., \& Santos, A. M. dos. (2019). Política de saúde LGBT e sua invisibilidade nas publicações em saúde coletiva. Saúde em Debate, 43(spe8), 305-323.

Brasil (2018). Edital $N^{o}$ 10, 23 de Julho 2018 Seleção para o Programa de Educação pelo Trabalho Para a Saúde Pet-Saúde/Interprofissionalidade 2018/2019. Brasília, DF: Ministério da Saúde; https://www.in.gov.br/materia/-/asset_publisher/Kujrw0TZC2Mb/content/id/33889041/do3-2018-07-24-editaln-10-23-de-julho-2018-selecao-para-o-programa-de-educacao-pelo-trabalho-para-a-saude-pet-saude-interprofissionalidade-2018-2019-33889037.

Ceccim, R. B. (2018). Conexões e fronteiras da interprofissionalidade: Forma e formação. Interface - Comunicação, Saúde, Educação, 22(suppl 2), 17391749 .

Costa, M. V. (2019). A educação interprofissional e o processo de formação em saúde no Brasil: pensando possibilidades para o futuro. Souza RMP, Costa PP, organizadores. Nova formação em saúde pública: aprendizado coletivo e lições compartilhadas na RedEscola. Fiocruz, 45-61.

Crisp, N., \& Chen, L. (2014). Global Supply of Health Professionals. New England Journal of Medicine, 370(10), 950-957.

Da Costa, L. D., Barros, A. D., de Jesus Prado, E. A., de Sousa, M. F., Cavadinha, E. T., \& Mendonça, A. V. M. (2017). Competência Cultural e Atenção à Saúde da população de lésbicas, gays, bissexuais travestis e transexuais (LGBT). Tempus Actas de Saúde Coletiva, 11(1), 105.

Estrela, C. (2018). Metodologia Científica: Ciência, Ensino, Pesquisa. Editora Artes Médicas. 
Farias, D. N. de, Ribeiro, K. S. Q. S., Anjos, U. U. dos, \& Brito, G. E. G. de. (2018). Interdisciplinaridade e interprofissionalidade na estratégia saúde da família. Trabalho, Educação e Saúde, 16(1), 141-162.

Frenk, J., Chen, L., Bhutta, Z. A., Cohen, J., Crisp, N., Evans, T., Fineberg, H., Garcia, P., Ke, Y., Kelley, P., Kistnasamy, B., Meleis, A., Naylor, D., PablosMendez, A., Reddy, S., Scrimshaw, S., Sepulveda, J., Serwadda, D., \& Zurayk, H. (2010). Health professionals for a new century: Transforming education to strengthen health systems in an interdependent world. The Lancet, 376(9756), 1923-1958.

Junqueira, R. D. (2007). Homofobia: limites e possibilidades de um conceito em meio a disputas. Bagoas-Estudos gays: gêneros e sexualidades, 1(01).

Koche, J. C. (2011). Fundamentos de metodologia científica. Petrópolis: Vozes. http://www.brunovivas.com/wp-content/uploads/sites/10/2018/ 07/K\%C3\%B6che-Jos\%C3\%A9-Carlos0D0AFundamentos-de-metodologia-cient\%C3\%ADfica-_teoria da0D0Aci\%C3\%AAncia-e-inicia\%C3\%A7\% C3\% A3 o-\%C3\%A0 pesquisa.pdfhttps://repositorio.ufsm.br/bitstream/handle/1/15824/Lic_Computacao_Metodologia-Pesquisa-Cientifica.pdf?sequence=1

Ludke, M. \& Andre, M. E. D. A. (2013). Pesquisas em educação: uma abordagem qualitativa. E.P.U.

Magnago, C., França, T., Belisário, S. A., \& Santos, M. R. dos. (2019). PET-Saúde/GraduaSUS na visão de atores do serviço e do ensino: Contribuições, limites e sugestões. Saúde em Debate, 43(spe1), 24-39.

Mello, L., Perilo, M., Braz, C. A. de, \& Pedrosa, C. (2011). Políticas de saúde para lésbicas, gays, bissexuais, travestis e transexuais no Brasil: Em busca de universalidade, integralidade e equidade. Sexualidad, Salud y Sociedad (Rio de Janeiro), 9, 7-28.

Organização Mundial da Saúde. (2010). Marco para ação em educação interprofissional e prática colaborativa. Editora. Freelance.

Pereira A. S. et al. (2018). Metodologia da pesquisa científica. UFSM.

Pereira, M. F. (2018). Interprofissionalidade e saúde: Conexões e fronteiras em transformação. Interface - Comunicação, Saúde, Educação, 22(suppl 2), 17531756

Prado, E. A. de J, \& de Sousa, M. F. (2017). Políticas Públicas e a saúde da população LGBT: uma revisão integrativa. Tempus - Actas De Saúde Coletiva, $11(1), 69-80$.

Rohrs, R. M. S., Dos Santos, C. F., Barbosa, R. D. S., Schulz, R. D. S., \& De Carvalho, M. B. (2017). Impacto da metodologia de simulação realística na graduação de enfermagem. Revista de Enfermagem UFPE on line, 11(12), 5269.

Santos, L. C. dos, Simonetti, J. P., \& Cyrino, A. P. (2018). A educação interprofissional na graduação de Medicina e Enfermagem em prática na atenção primária à saúde: A perspectiva dos estudantes. Interface - Comunicação, Saúde, Educação, 22(suppl 2), 1601-1611.

Silva, A. de C. A. da, Alcântara, A. M., Oliveira, D. C. de, \& Signorelli, M. C. (2020). Implementação da Política Nacional de Saúde Integral de Lésbicas, Gays, Bissexuais, Travestis e Transexuais (PNSI LGBT) no Paraná, Brasil. Interface - Comunicação, Saúde, Educação, 24, e190568.

Stephens, M., \& Ormandy, P. (2018). Extending conceptual understanding: How interprofessional education influences affective domain development. Journal of Interprofessional Care, 32(3), 348-357.

Supper, I., Catala, O., Lustman, M., Chemla, C., Bourgueil, Y., \& Letrilliart, L. (2014). Interprofessional collaboration in primary health care: A review of facilitators and barriers perceived by involved actors. Journal of Public Health, fdu102.

Vendruscolo, C., Silva, K. J. da, Durand, M. K., Metelski, F. K., \& Silva Filho, C. C. da. (2020). Ações do enfermeiro na interface com os núcleos ampliados de Saúde da Família e Atenção Básica. Revista da Escola de Enfermagem da USP, 54, e03642.

Vitiritti, B., Andrade, S. M. O., \& Peres, J. E. C. (2016). Diversidade sexual e relações profissionais: Concepções de médicos e enfermeiros. Temas em Psicologia, 24(4), 1389-1415. 